\title{
Assessment of Left Ventricular Function after Transcatheter Aortic Valve Implantation in Sever Aortic Stenosis: A Speckle Tracking Imaging Study
}

\author{
Ahmed S. Saad1', Said Shalaby Montaser², Ahmed M. Emara², Arif A. Al-Mulla³, Neveen I. Samy² \\ ${ }^{1}$ Shebin El Koum Teaching Hospital, Shebin El Koum, Egypt \\ ${ }^{2}$ Cardiovascular Disease Department, Faculty of Medicine, Menoufia University, Shebin El Koum, Egypt \\ ${ }^{3}$ Sheikh Khalifa Medical City, Abu Dhabi, UAE \\ Email: ahmedsaid9182@hotmail.com, *neveinsami@yahoo.com
}

How to cite this paper: Saad, A.S., Montaser, S.S., Emara, A.M., Al-Mulla, A.A. and Samy, N.I. (2020) Assessment of Left Ventricular Function after Transcatheter Aortic Valve Implantation in Sever Aortic Stenosis: A Speckle Tracking Imaging Study. World Journal of Cardiovascular Diseases, 10, 30-40.

https://doi.org/10.4236/wjcd.2020.101004

Received: October 23, 2019

Accepted: January 13, 2020

Published: January 16, 2020

Copyright $\odot 2020$ by author(s) and Scientific Research Publishing Inc. This work is licensed under the Creative Commons Attribution International License (CC BY 4.0).

http://creativecommons.org/licenses/by/4.0/ (c) (i) Open Access

\begin{abstract}
Background: Long standing aortic stenosis leads to elevated left ventricular (LV) pressure and as a result LV hypertrophy and myocardial fibrosis shall increase. The left ventricular ejection fraction (EF) usually remains adequately-preserved until advanced and late stages of aortic stenosis. But the preserved muscle of the LV is only limited to a portion of the entire myocardium. Speckle tracking echocardiography has proved its superiority to the standard two-dimensional echocardiography method in the detection of Left Ventricular (LV) function. Global Longitudinal strain (GLS) is considered as the most robust myocardial strain component. Objective: The aim of the study is to assess the early course of left ventricular reverse remodelling after Transcutaneous Aortic Valve Implantation (TAVI) in patients with symptomatic severe aortic valve stenosis. Methods. 50 patients with severe symptomatic valvular aortic stenosis undergoing TAVI as decided by the heart team after comprehensive discussion. Standard transthoracic echocardiography including Doppler analysis was performed. 2D speckle-tracking strain assessment of Global radial, circumferential and longitudinal strain at parasternal mid-ventricular short-axis view (at the level of papillary muscle) and from the apical long-axis, two-chamber and four-chamber views with a frame rate between 40 and 80 frames per second. Tracing of endocardial borders was done. Patients with significant coronary artery disease were fully revascularized by percutaneous coronary intervention prior to the study and the procedure. Results. 23 (46\%) patients were males, while 27 (54\%) were females. The patients' stratification according to comorbidities/associated risk factors re-
\end{abstract}


vealed that $54 \%$ of the patients had DM, $86 \%$ were hypertensive, $38 \%$ had chronic kidney disease (CKD), and $32 \%$ had a previous percutaneous coronary intervention (PCI). The mean age for our study participants ranged from 60 to 92 years (Mean $\pm S D=76.60 \pm 5.96$ ). Left ventricular diastolic diameter (LVDd) was $44.24 \pm 2.8 \mathrm{~mm}$ before TAVI that became $45.5 \pm 2.6 \mathrm{~mm}$ after TAVI, and ejection fraction (EF) increased from $52.82 \pm 6.3$ before TAVI to $56.70 \pm 5.4$ after TAVI, both with highly significant difference $(P<0.001)$, But there was not a significant difference regarding left ventricular systolic diameter (LVSd) after transcatheter aortic valve implantation (TAVI). Global longitudinal strain (GLS) improved from $-17.56 \pm 1.4$ to $-19.18 \pm 1.1$ with a $P$ value less than 0.001 . Global circumferential strain (GCS) also improved significantly from $-20.14 \pm 1.8$ before TAVI to $-21.72 \pm 1.7$ after TAVI with a $P$ value less than 0.001 . Global radial strain (GRS) also increased significantly from $37.38 \pm 8$ before TAVI to $41.68 \pm 6.3$ after TAVI with a $P$ value less than 0.001. Conclusion: TAVI is effective in improving left ventricular function presented not only by ejection fraction (EF\%), but also in global longitudinal strain (GLS), global circumferential strain (GCS), and global radial strain (GRS).

\section{Keywords}

Aortic Stenosis, Transcutaneous Aortic Valve Implantation, TAVI, Transcutaneous Aortic Valve Replacement, TAVR, Strain, Strain Rate, Speckle Tracking, Global Longitudinal Strain (GLS), Global Circumferential Strain (GCS), Global Radial Strain (GRS)

\section{Introduction}

Aortic valve stenosis (AS) is commonly found in elderly patients and it becomes associated with poor prognosis if not properly managed and treated as soon as possible [1]. Due to the prevalence of severe symptomatic AS in the geriatric population, AS presents usually with many co-morbidities, like hypertension, diabetes mellitus, and chronic kidney disease, which consequently increases the risk of cardiac surgery [1]. Transcatheter aortic valve implantation (TAVI) has established its effectiveness as a substitute to conventional surgery in patients with high grade aortic valve stenosis who have high cardiovascular surgery risk score [2]. TAVI can be performed using either self-expandable or balloon-expandable valved stents which would replace damaged and non-functioning aortic valves [3].

Long standing aortic stenosis leads to elevated left ventricular (LV) pressure and as a result LV hypertrophy and myocardial fibrosis shall increase. The left ventricular ejection fraction (EF) usually remains adequately-preserved until advanced and late stages of aortic stenosis. But the preserved muscle of the LV is only limited to a portion of the entire myocardium. It has been reported in many studies that the left ventricular myocardial long axis excursion, measured by 
M-mode echocardiography, is reduced in patients with severe aortic stenosis, despite that the heart has good ejection fraction [4].

While we are in need for more sensitive methods to detect myocardial deformation, tissue Doppler imaging and 2D speckle tracking analysis are highly sensitive markers for regional and global LV systolic function [5].

So, myocardial motion could be assessed by speckle tracking echocardiography which has proved its superiority to the standard two-dimensional echocardiography method in the detection of Left Ventricular (LV) function. After performing TAVI in patients with severe AS, an improvement was reported in numerous studies where echocardiography and echocardiographic strain analysis detected an improvement in left ventricular systolic and diastolic functions [6] [7].

Recently, published studies have demonstrated an improvement in LV systolic function assessed by tissue Doppler and speckle tracking strain imaging in patients with severe AS and preserved EF after conventional surgical aortic valve replacement in a mid-term and long-term follow up [8] [9] [10]. Global Longitudinal strain (GLS) is considered as the most robust myocardial strain component [11]. So this study aimed to assess the early course of left ventricular reverse remodelling after Transcutaneous Aortic Valve Implantation in patients with symptomatic severe aortic valve stenosis by speckle tracking imaging.

\section{Methods}

A prospective longitudinal study on 50 patients with severe symptomatic Valvular aortic stenosis during the period between June 2012 and July 2016 at Dubai Hospital, UAE.

Patients with symptomatic severe aortic valve stenosis and planned for TAVI as decided by the heart team after comprehensive discussion were included in this study. The operative risk of all patients was calculated according to the logistic European System for Cardiac Operative Risk Evaluation score. While Patients with inadequate recordings, Non-sinus rhythm such as atrial fibrillation, PreTAVI pacemaker, Patients who required post TAVI permanent pacing, Poor echocardiographic window and poor image quality, Bicuspid aortic valve undergoing TAVI, Post TAVI more than mild aortic regurgitation, Pre TAVI Severe mitral regurgitation of any cause were excluded from the study.

All included patients were subjected to pre and early ( 7 days) post procedure: Complete history taking, Thorough clinical evaluation, 12 lead resting ECG.

\subsection{Echocardiographic Examination}

The examinations were performed in the left lateral supine position with Vivid E9 scanner (GEVingmed Ulterasound).

Standard transthoracic echocardiography including Doppler analysis was performed according to the guidelines of the European Association of Cardiovascular Imaging and the American Society of Echocardiography [12] Patients were imaged in the left lateral decubitus position. LV volumes were measured 
and EF was calculated according to the modified Simpson's rule using the apical 4 and 2 chambers views.

Mean and maximum aortic valve pressure gradient were estimated by the modified Bernoulli equation and the flow velocity time integral over the ejection period on continuous wave Doppler recordings. The aortic valve area was determined by the continuity equation following the recommendations of the American Society of Echocardiography [12].

\subsection{D Speckle-Tracking Strain Analysis}

Assessment of radial, circumferential and longitudinal speckle tracking strain at parasternal mid-ventricular short axis view (at the level of papillary muscle) and from the apical long axis, two chamber and four chamber views with a frame rate between 40 and 80 frames per second (fps) were recorded for analysis.

Manual tracing of endocardial borders (the software automatically trace the region of interest including the entire myocardial wall) was done. In this process the left ventricle was divided into six segments.

Peak systolic longitudinal strain of the apical two, four chamber and long axis view was calculated averaging the peak systolic strain values of the six segments of the corresponding views. The global longitudinal peak systolic strain of the left ventricle was generated averaging peak systolic values of the three apical views. Peak systolic radial and circumferential strain was calculated averaging the values of the six LV segments from parasternal mid-ventricular short axis view as shown in Figure 1 \& Figure 2.

\subsection{Coronary Angiography and Interventions}

Patients with significant coronary artery disease were fully revascularized by percutaneous coronary intervention at least 2 weeks prior to the study and the procedure.

\subsection{The Statistical Analysis}

The collected data were tabulated and analyzed using the Statistical Package for Social Science (SPSS 20.0). The normality of distribution for the analyzed variables was tested using Kolmogorov-Smirnov test. The collected data were summarized in terms of mean \pm Standard Deviation (SD), for quantitative data and as number and percentage for qualitative data. The student $t$-test $(t)$ was used to compare differences in the mean value between two groups. While, the comparison between pre intervention and post intervention groups was tested using paired t-test. Correlation analysis to determine the association between variables was done, using Pearson correlation coefficient $(r)$. The accepted level of significance in this work was $0.05(P \leq 0.05)$.

\section{Results}

In Our Study, we had 50 patients with severe symptomatic aortic valve stenosis who underwent transcatheter aortic valve implantation (TAVI). 


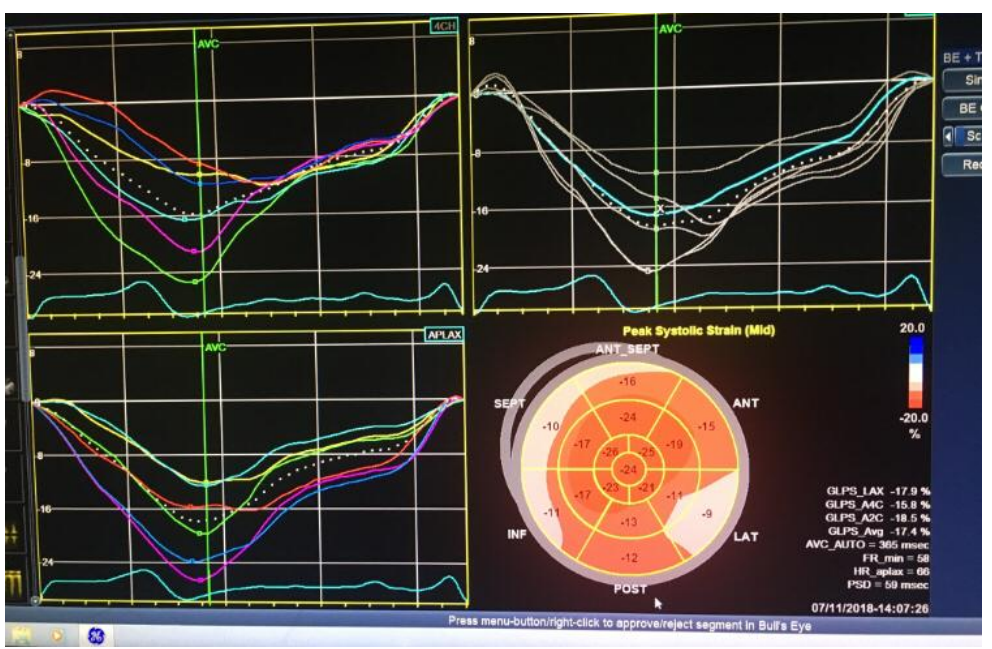

Figure 1. GLS before TAVI with value of $-17.9 \%$. (GLS: Global Longitudinal Strain, TAVI: Transcutaneous Aortic Valve Implantation).

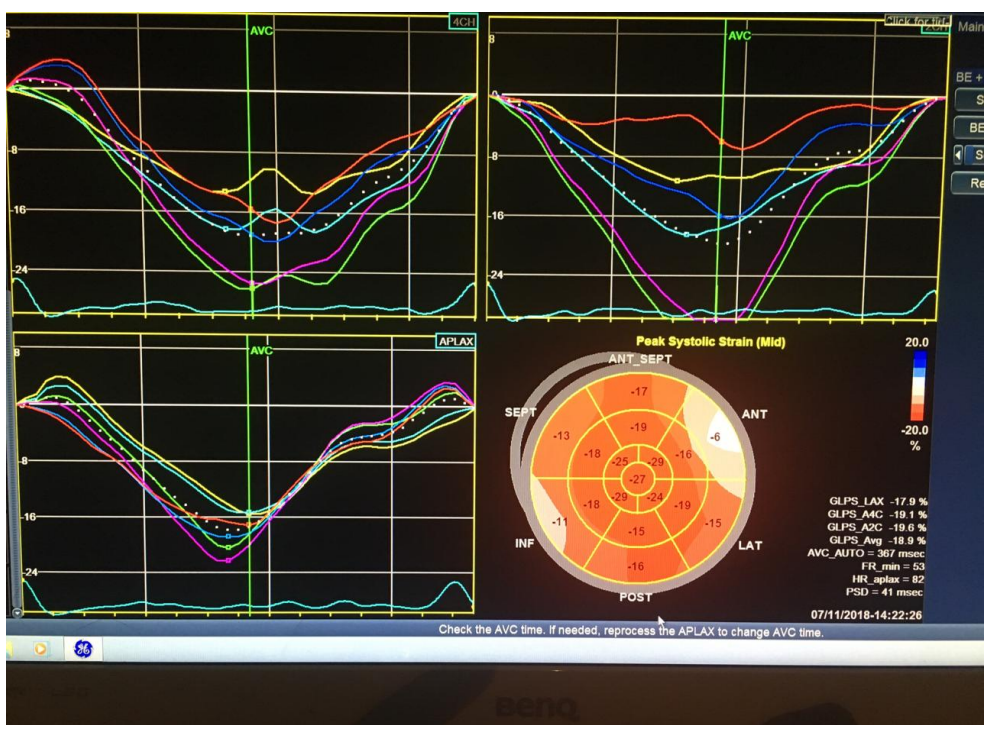

Figure 2. GLS after TAVI with value of $-18.9 \%$. (GLS: Global Longitudinal Strain, TAVI: Transcutaneous Aortic Valve Implantation).

Demographic criteria: From Our 50 patients, 23 (46\%) patients were males, while 27 (54\%) were females. The patients' stratification according to comorbidities/associated risk factors revealed that $54 \%$ of the patients had DM, $86 \%$ were hypertensive, $38 \%$ of them had chronic kidney disease (CKD), and $32 \%$ of these patients had a previous percutaneous coronary intervention (PCI) operation before. Distribution of Demographic Criteria among Study Population is demonstrated in Figure 3. The mean age for our study participants ranged from 60 to 92 years (Mean $\pm \mathrm{SD}=76.60 \pm 5.96$ ). Summaries for age and gender distribution can be visualised in Figure 4 and Figure 5.

\section{Echocardiographic Findings before and after TAVI}

Paired t-test results showed a highly significant difference after the operation 


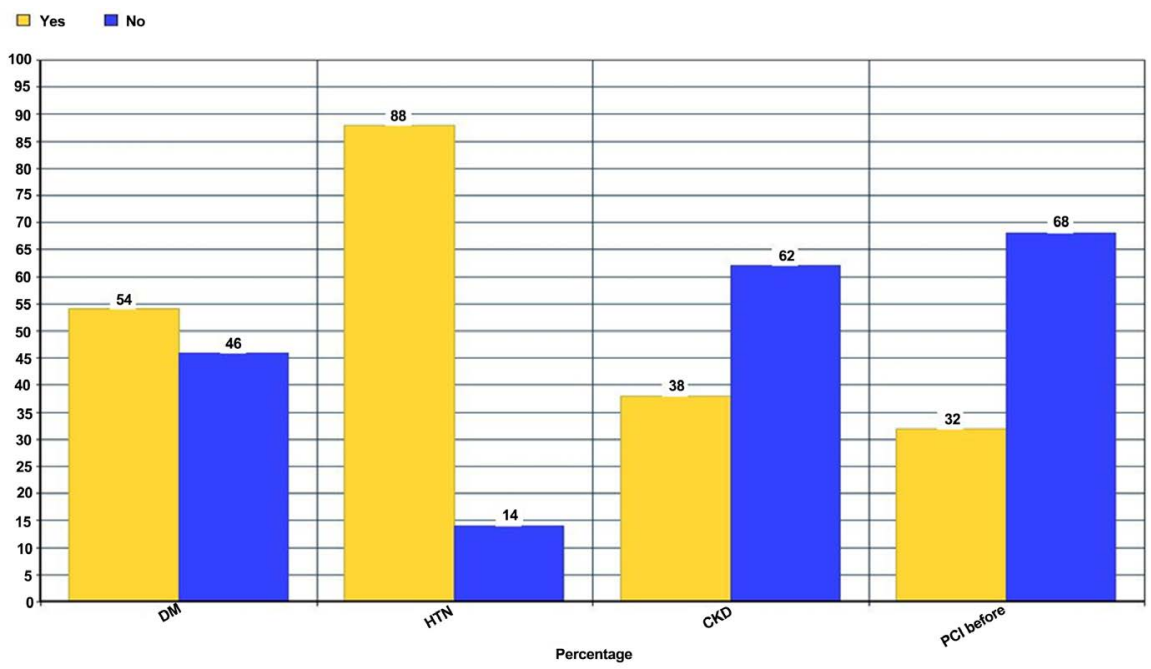

Figure 3. Distribution of demographic criteria among study population.

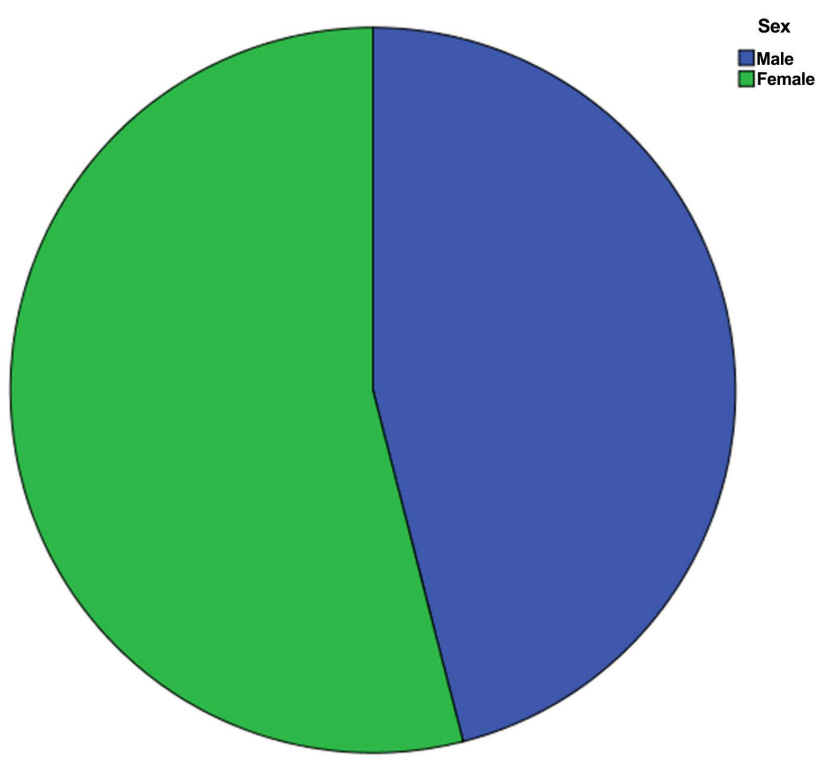

Figure 4. Male to female distribution $46 \%$ males, $54 \%$ females.

regarding the following parameters: left ventricular diastolic diameter (LVDd) was $44.24 \pm 2.8 \mathrm{~mm}$ before TAVI that became $45.5 \pm 2.6 \mathrm{~mm}$ after TAVI, and ejection fraction (EF) increased from $52.82 \pm 6.3$ before TAVI to $56.70 \pm 5.4$ after TAVI, both with highly significant difference $(P<0.001)$, But there was not a significant difference regarding left ventricular systolic diameter (LVSd) after transcatheter aortic valve implantation. Paired t-test results for LVDd, EF, and LVSd after the operation can be seen in Table 1 .

There was a statistically highly significant difference after TAVI operation in regard to global longitudinal strain (GLS) which improved from $-17.56 \pm 1.4$ to $-19.18 \pm 1.1$ with a $P$ value less than 0.001 . Global circumferential strain (GCS) also improved significantly from $-20.14 \pm 1.8$ before TAVI to $-21.72 \pm 1.7$ after TAVI with a $P$ value less than 0.001 . Global radial strain (GRS) also increased 


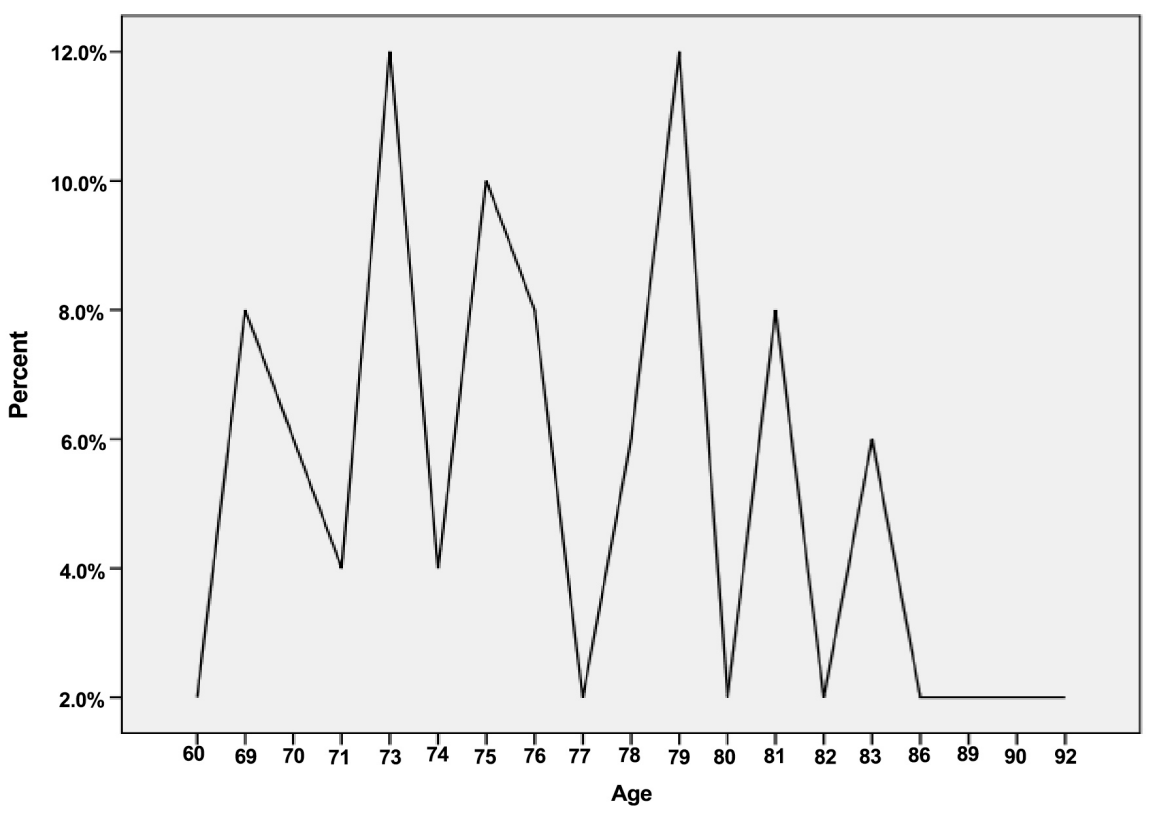

Figure 5. Distribution of age.

Table 1. Paired T test result for LVDd, EF, and LVSd.

\begin{tabular}{ccccc}
\hline \multirow{2}{*}{$\begin{array}{c}\text { Echo-cardiographic } \\
\text { Findings }\end{array}$} & Before & After & Paired T & \multirow{2}{\text{Test}}{} \\
\cline { 2 - 3 } & Mean \pm SD & Mean \pm SD & & \\
\hline LVDd (mm) & $44.24 \pm 2.8$ & $45.5 \pm 2.6$ & $\mathbf{5 . 3 5 5}$ & $<0.001$ \\
EF\% & $52.82 \pm 6.3$ & $56.70 \pm 5.4$ & $\mathbf{8 . 3 3}$ & $<0.001$ \\
LVSd (mm) & $30.46 \pm 1.9$ & $30.20 \pm 2$ & 1.24 & 0.221 \\
\hline
\end{tabular}

LVDd: Left Ventricular Diastolic diameter, EF: Ejection Fraction, LVSd: Left Ventricular Systolic diameter, m: millileter, SD: Standard Deviation.

significantly from $37.38 \pm 8$ before TAVI to $41.68 \pm 6.3$ after TAVI with a $P$ value less than 0.001. Paired t-test results for GLS, GCS, and GRS are demonstrated in Table 2 where $P$ was less than 0.001 .

\section{Discussion}

Transcatheter aortic valve implantation (TAVI) is a new, safe, and effective method that can be used to replace stenosed aortic valves in patients with high risk score for surgery [13].

The conventional method in monitoring viability and performance of the left ventricle during transcatheter aortic valve implantation is the two-dimensional echocardiography method. But recent studies have found that strain analysis-using speckle tracking approach-is superior to the routine two-dimensional echocardiography method [14].

Our results were in line with many studies that investigated the effect of TAVI on ejection fraction post-procedure. Lwin et al. [2] had 40 patients with significant aortic valve stenosis planned for transcatheter aortic valve implantation. 
Table 2. Paired T test result for GLS, GCS, and GRS.

\begin{tabular}{ccccc}
\hline \multirow{2}{*}{ Findings } & Before & After & Paired T Test & $\boldsymbol{P}$ \\
\cline { 2 - 5 } & Mean \pm SD & Mean \pm SD & & \\
\hline GLS & $-17.56 \pm 1.4$ & $-19.18 \pm 1.1$ & 9.59 & $<0.001$ \\
GCS & $-20.14 \pm 1.8$ & $-21.72 \pm 1.7$ & 7.07 & $<0.001$ \\
GRS & $37.38 \pm 8$ & $41.68 \pm 6.3$ & $\mathbf{8 . 5 1}$ & $<0.001$ \\
\hline
\end{tabular}

GLS: Global Longitudinal Strain, GCS Global Circumferential strain, GRS: Global Radial Strain, SD: Standard Deviation.

Their mean age was 85 years. They noticed a $4 \%$ improvement in left ventricular ejection fraction (LVEF) after TAVI operation. Also, D'Andrea et al. [15] analyzed the effect of TAVI on left ventricular (LV) and left atrial (LA) longitudinal function-assessed by speckle tracking echo-cardiographic-in patients with severe symptomatic aortic stenosis. They included 55 patients; whose mean age was $78.6 \pm 7.4$ years. Six months after the procedure, there found a significant improvement in ejection fraction $(P$ value $<0.0001)$. Additionally, Dimitriadis et al. [6] had 95 patients planned for TAVI who were followed up for three months. Patients were classified according to their ejection fraction. They found that TAVI resulted in improvement of left ventricular ejection fraction in patients with aortic stenosis and significant impairment in LVEF at baseline. Moreover, TAVI caused normalization of LVEF in patients with high/good EF at baseline. While our data were discordant with those of Grabskaya, E., et al. [7] who enrolled 40 patients with severe aortic stenosis. After six months follow up, left ventricular ejection fraction was not changed in a significant way after TAVI. Also, strain analysis values were not changed after the operation, but there was a significant change after a period of six months. In our study, there was a significant difference in the strain analysis measures after the operation namely: global longitudinal strain (GLS), global circumferential strain (GCS), and global radial strain (GRS) $(P$ value $<0.05)$. Our data were concordant with many studies which investigated the effect of TAVI on strain analysis measures. Naeim et al. [5] found a significant difference in global longitudinal strain has an improvement after TAVI in normal and depressed ejection fraction patients. They had 59 patients whose mean age was $80 \pm 11.7$ years. Transcatheter aortic valve implantation has benefited patients with $\mathrm{EF}$ more than or equal to $50 \%$ to improve their global circumferential strain which was impaired before the operation.

Moreover, Ahmed et al. [8] performed a similar study on 31 patients with severe aortic stenosis with high or prohibitive surgical risk score. The study implementers noticed a statistically significant change in global longitudinal strain after one and three days post TAVI. There was no significant difference in global longitudinal strain on the second day after the procedure. Furthermore, Luszczak et al. [9] found a significant improvement in global longitudinal strain (GLS) in aortic stenosis patients after transcatheter aortic valve implantation ( $P$ value $<0.001)$. They included 49 patients with moderate to severe aortic stenosis 
and left ventricular ejection fraction more than or equal to $50 \%$. They noticed a significant decrease in GLS in symptomatic AS patients compared to the asymptomatic group $(P$ value $=0.02)$. Besides, Marwan et al. [10] found that global longitudinal strain was significantly lower after transcatheter aortic valve replacement operation compared to its baseline value. They included 25 patients with severe symptomatic aortic stenosis. Their mean age was $78 \pm 9$ years, and 13 of them were males. Additionally, D'Andrea et al. [15] found a significant improvement of left ventricular global longitudinal-measured by speckle tracking-after transcatheter aortic valve implantation. Also, Grabskaya et al. [7] found a significant effect of TAVI on strain measures. They enrolled 36 patients with aortic stenosis scheduled for transcutaneous aortic valve replacement. Their mean age was $83 \pm$ 6 years. They found a significant decrease in global longitudinal strain measure one month after the operation. There were no significant changes in global circumferential and global radial strain measurements after TAVI. In their study, they hypothesized that myocardial deformation was not influenced by pacemaker implantation or the duration of left bundle branch block. Similarly, Alenezi et al. [14] found a significant change in global longitudinal strain after transcatheter aortic valve replacement $(P$ value $<0.001)$.

\section{Limitations}

Short time of follow up after the procedure. Image quality of echocardiographic study in elderly patients can sometimes be unclear. This is because of echocardiography's limited planes, acoustic shadowing, and certain patient related conditions.

\section{Conclusion}

Transcatheter aortic valve implantation (TAVI) is an effective and safe alternative to open heart surgery that can be used to replace the aortic valve in patients with high risk through improving left ventricular function presented not only by ejection fraction (EF), but also in global longitudinal strain (GLS), global circumferential strain (GCS), and global radial strain (GRS) measures.

\section{Conflicts of Interest}

The authors declare no conflicts of interest regarding the publication of this paper.

\section{References}

[1] Iung, B. (2003) A Prospective Survey of Patients with Valvular Heart Disease in Europe: The Euro Heart Survey on Valvular Heart Disease. European Heart Journal, 24, 1231-1243. http://eurheartj.oxfordjournals.org/content/24/13/1231.long https://doi.org/10.1016/S0195-668X(03)00201-X

[2] Lwin, M., Humphries, J., Challa, A., Walters, D., Lau, K., Koitka, K., et al. (2018) Global Longitudinal Strain in Patients with Severe Aortic Stenosis Undergoing Transcatheter Aortic Valve Implantation. Heart, Lung and Circulation, 27, S241. https://doi.org/10.1016/j.hlc.2018.06.446 
[3] ElBardissi, A.W., Shekar, P., Couper, G.S., and Cohn, L.H. (2011) Minimally Invasive Aortic Valve Replacement in Octogenarian, High-Risk, Transcatheter Aortic Valve Implantation Candidates. The Journal of Thoracic and Cardiovascular Surgery, 141, 328-335. https://doi.org/10.1016/j.jtcvs.2010.08.056

[4] Kupari, M., Turto, H. and Lommi, J. (2005) Left Ventricular Hypertrophy in Aortic Valve Stenosis: Preventive or Promotive of Systolic Dysfunction and Heart Failure? European Heart Journal, 26, 1790-1796. https://doi.org/10.1093/eurheartj/ehi290

[5] Naeim, H., Alattawi, F.O., Al Amodi, O., Al Ghamdi, S., Alharbi, I.H., Qanitha, S., et al. (2018) Echo after Transcatheter Aortic Valve Implantation (TAVI) in Medina Cardiac Centre. Journal of the Saudi Heart Association, 30, 356.

https://doi.org/10.1016/j.jsha.2018.05.004

[6] Dimitriadis, Z., Scholtz, S., Ensminger, S., Wiemer, M., Fischbach, T., Scholtz, W., et al. (2017) Left Ventricular Adaptation after TAVI Evaluated by Conventional and Speckle-Tracking Echocardiography. International Journal of Cardiology, 228, 633-637. https://doi.org/10.1016/j.ijcard.2016.11.035

[7] Grabskaya, E., Becker, M., Altiok, E., Dohmen, G., Brehmer, K., Hamada-Langer, S., et al. (2011) Impact of Transcutaneous Aortic Valve Implantation on Myocardial Deformation. Echocardiography, 28, 397-401. https://doi.org/10.1111/j.1540-8175.2010.01378.x

[8] Ahmed, D., Barin, E., Ng, M., Coelho, B., Foy-Moxey, C. and Hammond, R. (2018) Analysis of Improvement in Myocardial Strain Early After Transcatheter Aortic Valve Implantation. Heart, Lung and Circulation, 27, S216.

https://linkinghub.elsevier.com/retrieve/pii/S1443950618311223 https://doi.org/10.1016/j.hlc.2018.06.391

[9] Luszczak, J., Olszowska, M., Drapisz, S., Plazak, W., Karch, I., Komar, M., et al. (2012) Assessment of Left Ventricle Function in Patients with Symptomatic and Asymptomatic Aortic Stenosis by 2-Dimensional Speckle-Tracking Imaging. Medical Science Monitor, 18, MT91-MT96. https://doi.org/10.12659/MSM.883587

[10] Marwan, M., Ammon, F., Bittner, D., Röther, J., Mekkhala, N., Hell, M., et al. (2018) CT-Derived Left Ventricular Global Strain in Aortic Valve Stenosis Patients: A comparative Analysis Pre and Post Transcatheter Aortic Valve Implantation. Journal of Cardiovascular Computed Tomography, 12, 240-244.

https://doi.org/10.1016/j.jcct.2018.01.010

[11] Leitman, M., Lysyansky, P., Sidenko, S., et al. (2004) Two-Dimensional Strain-a Novel Software for Real Time Quantitative Echocardiographic Assessment of Myocardial Function. Journal of the American Society of Echocardiography, 17, 1021-1029.

[12] Baumgartner, H., Hung, J., Bermejo, J., Chambers, J.B., Edvardsen, T., Goldstein, S., Lancellotti, P., LeFevre, M., Miller Jr., F. and Otto, C.M. (2017) Recommendations on the Echocardiographic Assessment of Aortic Valve Stenosis: A Focused Update from the European Association of Cardiovascular Imaging and the American Society of Echocardiography. European Heart Journal-Cardiovascular Imaging, 18, 254-275. https://doi.org/10.1093/ehjci/jew335

[13] Lefevre, T., Kappetein, A.P., Wolner, E., Nataf, P., Thomas, M., et al. (2011) One Year Follow-up of the Multi-Centre European PARTNER Transcatheter Heart Valve Study. European Heart Journal, 32, 148-157. https://doi.org/10.1093/eurheartj/ehq427

[14] Alenezi, F., Fudim, M., Rymer, J., Dunning, A., Chiswell, K., Swaminathan, M., et al. (2019) Predictors and Changes in Cardiac Hemodynamics and Geometry with Transcatheter Aortic Valve Implantation. The American Journal of Cardiology, 
123, 813-819. https://doi.org/10.1016/j.amjcard.2018.11.038

[15] D’Andrea, A., Padalino, R., Cocchia, R., Di Palma, E., Riegler, L., Scarafile, R., et al. (2015) Effects of Transcatheter Aortic Valve Implantation on Left Ventricular and Left Atrial Morphology and Function. Echocardiography, 32, 928-936. https://doi.org/10.1111/echo.12808 